\title{
A MATHEMATICAL MODEL FOR INSTABILITY IN SNOW GLIDING MOTION
}

\author{
by
}

\author{
Yasuaki Nohguchi*
}

(Versuchsanstalt für Wasserbau, Hydrologie und Glaziologie, ETH, CH-8092 Zürich, Switzerland)

\section{ABSTRACT}

In this paper a fundamental model is proposed to describe the time-dependent behaviour of uniform snow glide on an infinite slope. This model is composed both of a mechanical balance equation relating a driving force and a resistant force, and of a rate equation for a real contact area at the boundary. In the model, a steady motion of glide is described as a stable equilibrium of glide motion, and a non-steady motion is considered as a transient one not in equilibrium. Finally, the model is applied to a full-depth avalanche release caused by acceleration of snow glide. By comparing the model predictions with glidevelocity field data, a proper model of acceleration prior to full-depth avalanche release is determined. As a result, we can obtain the safety standards to be applied in the event of full-depth avalanches in terms of a glide velocity.

\section{INTRODUCTION}

Snow glide is a quasi-static movement of a snow cover on a slope; therefore, it is mechanically in balance even though the glide velocity is not necessarily steady. The acceleration of the snow-glide velocity before a full-depth avalanche release is a typical example of non-steady gliding motion in Nature (Nohguchi and others, 1986). This suggests that the steady motion of snow glide is a particular state in a time-dependent frame (Fig. 1) and does not pre-exist. In this view, both the state of rest and that of a full-depth avalanche release are also particular states in non-steady glide motion.

Physical studies of snow-glide motion have been carried out by In der Gand and Zupancix (1966), Salm (1977), McClung (1981), and Lackinger (1987), and these have been reviewed by McClung (1987). Their work deals mainly with a steady motion of snow glide. In contrast, on the other hand, Endo $(1983,1985)$ has introduced a physical process including time to explain the mechanism of the acceleration to avalanche release on a slope covered by bamboo bush.

In this paper, we consider steady glide motion, rest, and full-depth avalanche release as particular states in time-dependent glide motion, as shown in Figure 1. From this standpoint, a fundamental model is proposed which will describe time-dependent behaviour of snow glide. The model has the flexibility to represent different physical processes at the interface between snow and the ground, because the physical process is not uniquely defined by details of difference in these boundary conditions, such as the presence and nature of vegetation on the ground.

* Present address: Nagaoka Institute of Snow and Ice Studies, National Research Center for Disaster Prevention, Suyoshi, Nagaoka-shi 940 , Japan.

\section{A TIME-DEPENDENT MODEL OF BASAL RESISTANCE} FORCE

The time variation in glide velocity during a process of acceleration is considered as continuous so long as we see only a particular time-scale. Such a time-scale may range from minutes on a grass slope (Nakamura and others, 1972) to days on a slope of low trees (Nohguchi and others, 1986), according to the physical processes at the interface arising from the differences in vegetation. This indicates that the process of non-steady motion should be described by the continuous variation of a specific internal variable, even though the initiation of non-steady motion arises from changes in external conditions such as air temperature, snowfall, or rainfall. Nohguchi (1983) has introduced the concept of real contact area as an internal variable to describe directly the state of contact between snow and ground, making the analogy with the adhesion theory on friction of ice proposed by Tsushima (1977). Using this real contact area, $A$, the resistance force, $R$, against a snow cover with real contact area $A$ and velocity $v$ relative to the ground, can be generally described as

$$
R=R(A, v)
$$

where $A$ is not necessarily constant, except at equilibrium. With $R$ so defined, we take the rate of the change of $A$ to be given by

$$
\mathrm{d} A / \mathrm{d} t=-p(A, v)+q(A, v)
$$

where $p$ is the rate of the decrease in size of the real contact area due to shear, and $q$ is the rate of the increase in contact-area size due to creation of new real contact area.

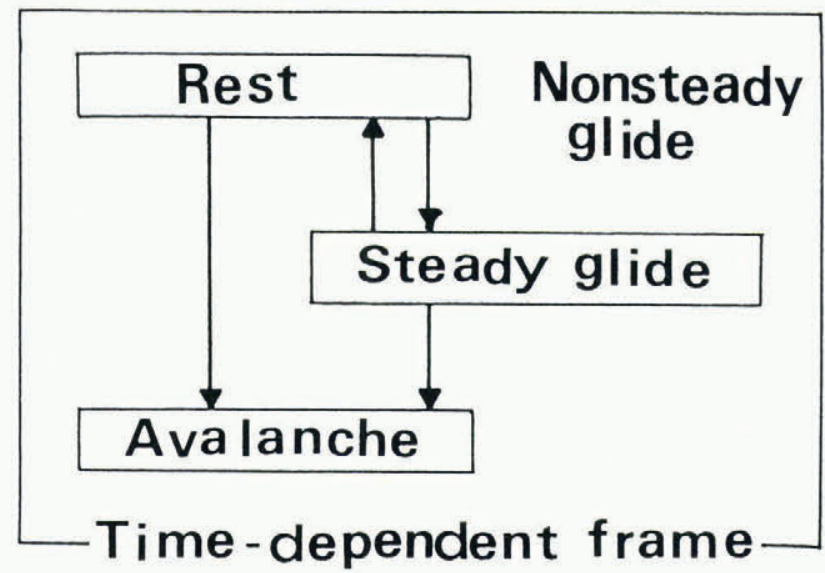

Fig. 1. Structure of the time-dependent model. 

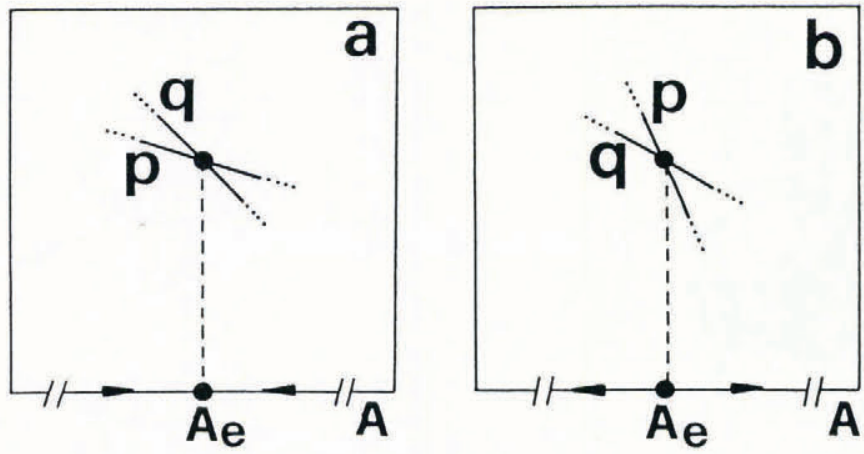

Fig. 2. Steady-state stability; unstable equilibrium.

If $p$ is equal to $q$, then the snow-glide motion is in an equilibrium condition. On the other hand, if $p$ is not equal to $q$, the real contact area and glide-velocity change with time; therefore, the snow-glide motion is not in equilibrium. In this model, the physical process is expressed in terms of the functions $p, q$, and $R$.

\section{GOVERNING EQUATIONS FOR SNOW GLIDE}

In a general glide problem, the resistance-force model represents the boundary condition at the bed surface for the mechanical balance equation of snow cover. For simplicity, we consider a rigid-body motion for snow cover without deformation on an infinite slope. The governing equations then are

$$
\begin{gathered}
f=R(A, v) \\
\mathrm{d} A / \mathrm{d} t=-p(A, v)+q(A, v)
\end{gathered}
$$

where $f$ is a driving force due to gravity which is generally a function of time.

When $f$ remains constant and the following equations

$$
\begin{gathered}
f=R\left(A_{\mathrm{e}}, v_{\mathrm{e}}\right) \\
p\left(A_{\mathrm{e}}, v_{\mathrm{e}}\right)=q\left(A_{\mathrm{e}}, v_{\mathrm{e}}\right)
\end{gathered}
$$

can be solved, their solutions are the equilibrium values.

For $v=v_{\mathrm{e}}$ and $A=A_{\mathrm{e}}$, the glide velocity and the state of contact are steady because in such conditions $\mathrm{d} A / \mathrm{d} t=0$.

$p$ and $q$ can be represented only by $A$ because $v$ can be described by $A$ from Equation (3). When $p$ and $q$, the functions of $A$, have the relationship shown in Figure 2a, the equilibrium state is stable, that is, steady snow glide is stable. On the other hand, in the case shown in Figure $2 \mathrm{~b}$, when the equilbrium state is unstable, steady snow glide cannot exist stably.

\section{EXAMPLE OF SNOW-GLIDE BEHAVIOUR}

We consider the following process as a simple example:

$$
\begin{gathered}
p=a v A \\
q=b\left(A_{\infty}-A\right) l\left(A_{\infty}-A\right) \\
R=\left(\varepsilon_{0}+\varepsilon_{1} v\right) A
\end{gathered}
$$

where $l(x)$ is a step function defined by

$$
l(x)= \begin{cases}1 & x \geqslant 0 \\ 0 & x<0,\end{cases}
$$

and $a, b, A_{\infty}, \quad \varepsilon_{0}$, and $\varepsilon_{1}$ are external parameters independent of $A$ and $v$. Through these relationships, we assume that the rate of decrease of $A, p$, is proportional to the glide velocity, $v$, and real contact area, $A$, such that $p$ is equal to zero for $v=0$ or $A=0$. The rate of increase of $A, q$, is assumed to be proportional to $A_{\infty}-A$ for $A \leqslant A_{\infty}$. Therefore, $A_{\infty}$ is the value of $A$ in equilibrium for $v=0$. The value of $q$ is then proportional to deviation of $A$ from its equilibrium value, $A_{\infty}$. This process represents the increase of real contact area due to viscous deformation of the snow. The resistance force, $R$, is assumed to be proportional to the real contact area such that $R$ is an increasing function of $A$. Then $\left(\varepsilon_{0}+\varepsilon_{1} v\right)$ can be regarded as a shear strength which depends on velocity.

By substituting Equations (7), (8), and (9) into Equations (3) and (4), we can obtain non-dimensional equations for snow glide as follows

$$
\mathrm{d} A^{*} / \mathrm{d} t^{*}=-a^{*} v^{*} A^{*} l\left(f^{*}-A^{*}\right)+\left(1-A^{*}\right) l\left(1-A^{*}\right)
$$

$$
f^{*}=\left(1+v^{*}\right) A^{*}
$$

where $a^{*}=a \varepsilon_{0} / b \varepsilon_{1} ; \quad f^{*}=f /\left(\varepsilon_{0} A_{\infty}\right) ; \quad t^{*}=b t ; \quad v^{*}=\varepsilon_{1} / \varepsilon_{0} v_{;}$ $A^{*}=A / A_{\infty}$. From the mechanical balance Equation (12), the velocity can be represented in terms of the real contact area as

$$
v^{*}=\left(f^{*}-A^{*}\right) l\left(f^{*}-A^{*}\right) / A^{*} .
$$

By substituting Equation (13) into the rate Equation (11), the equation for $A$ can be obtained as

$$
\mathrm{d} A^{*} / \mathrm{d} t^{*}=-a^{*}\left(f^{*}-A^{*}\right) l\left(f^{*}-A^{*}\right)+\left(1-A^{*}\right) l\left(1-A^{*}\right) .
$$

Thus, the behaviour of snow glide is described through the time variation of the real contact area. For $A^{*}=0, v^{*}$ has an infinite velocity in a quasi-static sense; therefore, when $A^{*}=0$ this indicates the release of a full-depth avalanche On the other hand, for $A^{*}>f^{*}$, the velocity $v^{*}=0$, and so the material is in a resting state. Equation (14) is governed by two non-dimensional parameters, $a^{*}$ and $f^{*}$ These are external factors which are dependent on slope angle, temperature, water content, weight of snow cover, and other characteristics, all of which change with time.

When external conditions remain constant, that is when $a^{*}$ and $f^{*}$ are constant, the behaviour of the solutions for Equation (14) can be classified into the following four types, according to the values of $a^{*}$ and $f^{*}$ (Figs 3 and 4):

Type $1\left(f^{*}<1\right.$ and $\left.f^{*} a^{*}<1\right)$

Any gliding motion decelerates to rest. Therefore, the state of rest is the stable state and a full-depth avalanche cannot occur for any perturbation.

Type $2\left(f^{*}>1\right.$ and $\left.f^{*} a^{*}<1\right)$

A stable steady glide motion exists for any perturbation and a full-depth avalanche cannot occur.

Type $3\left(f^{*}<1\right.$ and $\left.f^{*} a^{*}>1\right)$

A single non-stable equilibrium state exists. If the snow cover has a glide velocity greater than $v_{\mathrm{e}}^{*}=\left(f^{*}-1\right) /$ $\left(1-f^{*} a^{*}\right)$, it accelerates to form an avalanche. On the other hand, if the glide velocity is lower than this value, it decelerates to rest.

Type $4\left(f^{*}>1\right.$ and $\left.f^{*} a^{*}>1\right)$

Any gliding motion with these conditions accelerates to produce an avalanche. Therefore, only a full-depth avalanche release will enable the snow cover on such a slope to become stable.

In type 1 and 2 categories it is impossible to release a full-depth avalanche whatever the perturbation. However, in type 3 conditions, such a release is possible because a state of rest and a state of acceleration to produce an avalanche are both possible under the specified condition. In type 3 snow cover, once the snow cover has gained a glide velocity greater than $v_{e}$, as the result of some perturbation such as an artificial explosion or water supply, it will accelerate until a full-depth avalanche is released. As a 


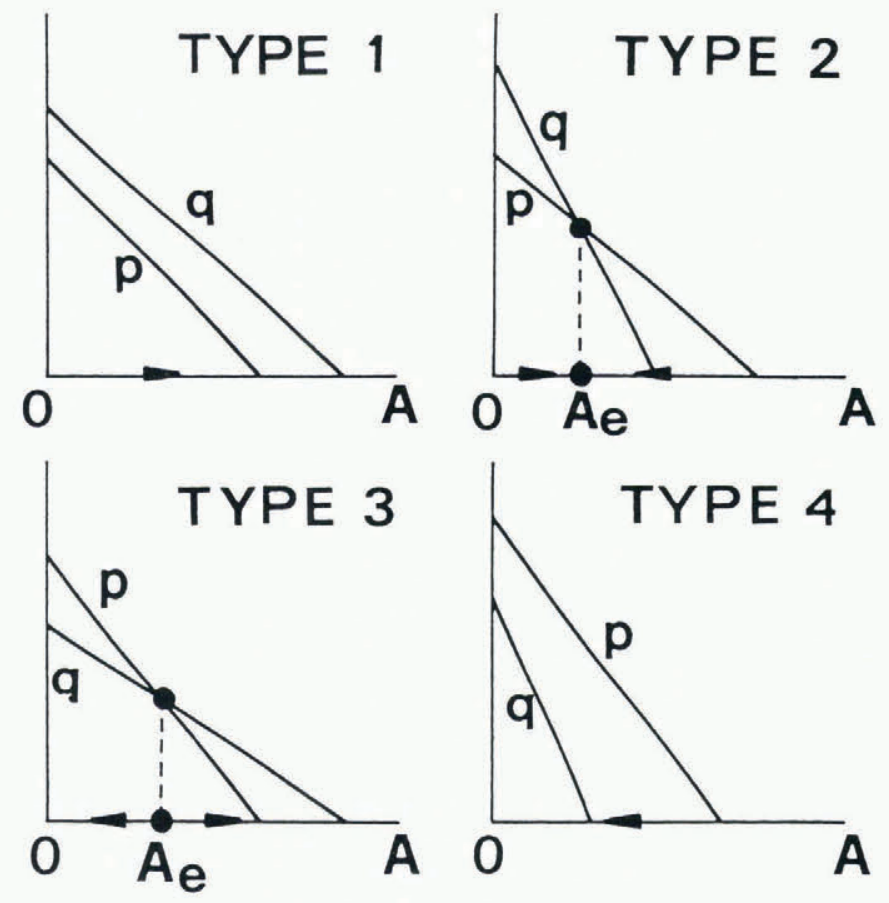

Fig. 3. Four types of behaviour derived by solving Equation (14).

result of changes in external conditions, snow cover classified as one type may sometimes change into another category. For example, if the external conditions change from those specified for type 1 to those specified for type 2 , the snow cover at rest will begin to move at a steady velocity. Moreover, changes in $f^{*}$ and $a^{*}$ values from those of type 2 to those of type 4 will result in snow cover with a steady velocity accelerating to form a full-depth avalanche.

In general, the characteristics of snow-glide behaviour which are due to changes in external conditions depend on the careful selection of the functions $p, q$, and $R$ (Nohguchi, 1983). Therefore, for the most appropriate selection of $p, q$, and $R$ according to each physical process at the snow-cover boundary, qualitative comparisons with field data are essential.

\section{ACCELERATION OF SNOW GLIDE AND AVALANCHE} RELEASE

Here, we apply the model only to the acceleration of snow glide before a full-depth avalanche release. In such a case, we may assume that the rate of decrease in $A$ is much greater than the rate of increase. By neglecting $q$ in Equation (4), we then obtain

$$
\begin{gathered}
\mathrm{d} A / \mathrm{d} t=-p(A, v) \\
f=R(A, v) .
\end{gathered}
$$

If the physical processes represented by Equations (7) and (9) are introduced into Equations (15) and (16), the glide velocity, $v$, is governed by the following equation

$$
\mathrm{d} v / \mathrm{d} t=a \varepsilon_{0} / \varepsilon_{1}\left(1+\varepsilon_{1} / \varepsilon_{0} v\right) v
$$

and this equation indicates that the accleration of the snow glide is an increasing function of the snow-glide velocity itself. From Equation (17), the time to avalanche release is given by

$$
T_{\mathrm{C}}=\frac{\varepsilon_{1}}{\mathrm{a} \varepsilon_{0}} \log \frac{\varepsilon_{0} / \varepsilon_{1}+v_{0}}{v_{0}}
$$

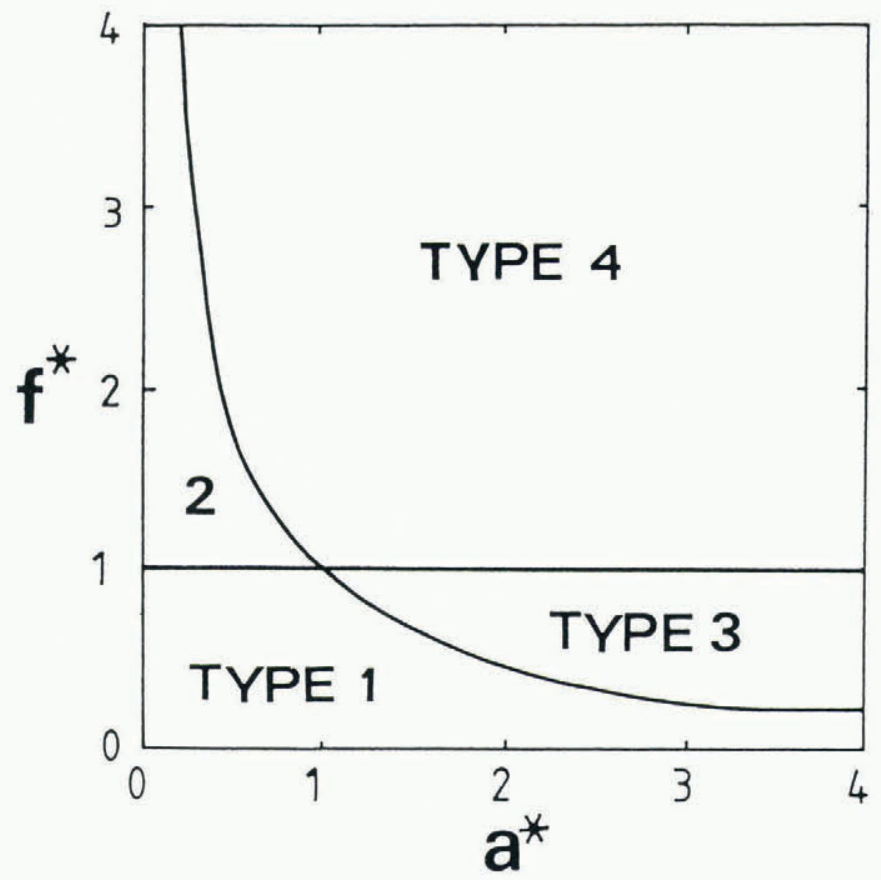

Fig. 4. Representation of behaviour types in parameter space.

where $T_{c}$ is time to an avalanche release for a snow cover with the glide velocity $v=v_{0}$. When the velocity is sufficiently large, Equations (17) and (18) can be approximated as

$$
\begin{aligned}
& \mathrm{d} v / \mathrm{d} t=a v^{2} \\
& T_{\mathrm{c}}=1 /\left(a v_{0}\right) .
\end{aligned}
$$

We now compare the concrete model, described by Equations (15) and (16) together with the physical process in Equations (7) and (9), with field data on glide velocity. If the field data can be matched with the theoretical results, we can use them to obtain unknown parameters as required. On the other hand, if the field data cannot be explained by reference to the theoretical results, other physical processes must be considered.

We have four sets of field data for snow glide involving continuous acceleration to an avalanche release or to a small break-down of snow cover (Nohguchi and others, 1986). Three sets of data were obtained on natural slopes with low trees (S-1, S-2, S-3) at Hosono, Japan, by use of a gear-type glide meter; the fourth set of data was obtained on an experimental slope with short-cut grass $(\mathrm{T}-1)$ at Tokamachi, Japan, by using a glide shoe. Figure 5 shows that the field data obtained give a reasonable fit with the theoretical curves derived from Equations (17) and (18). By this fit, the two parameters $a$ and $\varepsilon_{1} / \varepsilon_{0}$ can be found (Table I).

Full-depth avalanches frequently occur in Niigata Prefecture, Japan, on the low tree slopes. Therefore, using the above results and Equation (20), we propose the following safety measures against full-depth avalanches on slopes covered by low trees. In order to achieve safety, we estimate the parameter $a$ at $10 \mathrm{~m}^{-1}$ for a low tree-covered slope, because $T_{c}$ is small for large values of $a$. Then, if the glide velocity is $10 \mathrm{~mm} / \mathrm{h}$, which is of the same order of magnitude as the characteristic velocity, $T_{\mathrm{c}}$ becomes $10 \mathrm{~h}$; we call this value the caution velocity, because $10 \mathrm{~h}$ is long enough to escape. However, if the glide velocity is of the order of $10 \mathrm{~mm} / \mathrm{min}, T_{\mathrm{c}}$ becomes $10 \mathrm{~min}$; we call this the danger velocity, because $10 \mathrm{~min}$ is a short time in which to escape. In general, for a steady glide motion, a typical value for the glide velocity is the order of $10 \mathrm{~mm} / \mathrm{d}$ (de Quervain, 1966). We summarize the approximate safety standards for use in full-depth avalanches on a slope covered by low trees in Table II. 

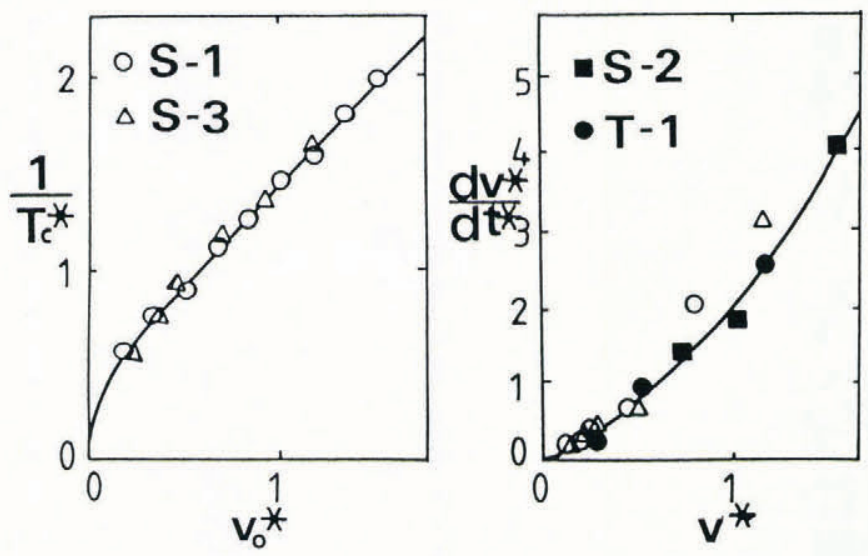

Fig. 5. Theoretical curves matched with field data. (a) Glide velocity $\left(v_{0}^{*}=\left(\varepsilon_{1} / \varepsilon_{0}\right) v_{0}\right)$ against reciprocal of time $\left(T_{\mathrm{c}}^{*}=\left(a \varepsilon_{0} / \varepsilon_{1}\right) T_{\mathrm{c}}\right)$. (b) Glide velocity against acceleration $\left(\mathrm{d} v^{*} / \mathrm{d} t=\left(a \varepsilon_{0} / \varepsilon_{1}\right) \mathrm{d} v / \mathrm{d} t\right)$.

TABLE I. SUMMARY OF PARAMETERS. $\left(T=\varepsilon_{1} /\left(a \varepsilon_{0}\right)\right.$, $V=\varepsilon_{0} / \varepsilon_{1}$, AND $L=T V=a^{-1}$ ARE CHARACTERISTIC TIME, VELOCITY, AND LENGTH, RESPECTIVELY.)

\begin{tabular}{lcrrll} 
Site & \multicolumn{1}{c}{$a$} & $\varepsilon_{1} / \varepsilon_{0}$ & $T$ & $V$ & $L$ \\
& $\left(\mathrm{~m}^{-1}\right)$ & $(\mathrm{h} / \mathrm{m})$ & $(\mathrm{h})$ & $(\mathrm{mm} / \mathrm{s})$ & $(\mathrm{m})$ \\
& & & & & \\
$\mathrm{S}-1$ & 0.92 & 8.3 & 9.1 & 0.033 & 1.1 \\
$\mathrm{~S}-2$ & 2.2 & 34.0 & 16.0 & 0.0081 & 0.46 \\
$\mathrm{~S}-3$ & 5.6 & 23.0 & 4.2 & 0.012 & 0.18 \\
$\mathrm{~T}-1$ & 3.3 & 210.0 & 64.0 & 0.0013 & 0.31
\end{tabular}

TABLE II. GLIDE VELOCITY AND SAFETY STANDARDS APPROPRIATE IN A FULL-DEPTH AVALANCHE RELEASE ON A SLOPE COVERED BY LOW TREES

$\begin{array}{llc}\text { Velocity } & T_{\mathrm{c}} & \text { Safety } \\ 10 \mathrm{~mm} / \mathrm{m} & 10 \mathrm{~min} & \text { danger } \\ 10 \mathrm{~mm} / \mathrm{h} & 10 \mathrm{~h} & \text { caution } \\ 10 \mathrm{~mm} / \mathrm{d} & - & \text { safe }\end{array}$

\section{CONCLUDING REMARKS}

In order qualitatively to understand the whole behaviour of snow glide, including non-steady motion, it is necessary to make use of the systematic framework of a mathematical model, which is required to be capable of analysing the results of observations and/or experiments. In this paper we have considered steady glide motion, rest, and full-depth avalanche release as particular states in time-dependent glide motion. Using this view, a fundamental model has been proposed to describe the time-dependent behaviour of snow glide. From this model, the inter-relation between the states of steady glide, rest, and acceleration to production of an avalanche has been made clear for a simple example of these physical processes. Finally, the model was applied to the acceleration before a full-depth avalanche release, and by comparison with field data the safety standards appropriate for a full-depth avalanche on a slope covered by low trees were estimated in terms of glide velocity.

\section{REFERENCES}

Endo, Y. 1983. Glide processes of a snow cover as a release mechanism of an avalanche slope covered with bamboo bushes. Contrib. Inst. Low Temp. Sci., Ser. A, $32,39-68$.

Endo, Y. 1985. Release mechanism of an avalanche on a slope covered with bamboo bushes. Ann. Glaciol., 6, 256-257.

Gand, H.R. In der and M. Zupaň̌ ix. 1966. Snow gliding and avalanches. International Association of Scientific Hydrology Publication 69 (Symposium at Davos 1965 Avalanches and Physics of Snow), 230-242.

Lackinger, B. 1987. Stability and fracture of the snow pack for glide avalanches. International Association of Hydrological Sciences Publication 162 (Symposium at Davos 1986 - Avalanche Formation, Movement and Effects), 229-241.

McClung, D.M. 1981. A physical theory of snow gliding. Can. Geotech. J., 18(1), 86-94.

McClung, D.M. 1987. Mechanics of snow slab failure from a geotechnical perspective. International Association of Hydrological Sciences Publication 162 (Symposium at Davos 1986 - Avalanche Formation, Movement and Effects), 475-508.

Nakamura, T and Y. Yamada. 1972. A small avalanche release over an experimental slope artificially covered with long grass. In 1972 Symposium of the Japanese Society of Snow and Ice. [In Japanese.]

Nohguchi, Y. 1983. Mathematical model of snow gliding. Rep. Natl. Res. Cent. Disaster Prevention, 30, 189-206. [In Japanese.]

Nohguchi, Y., Y. Yamada, and T. Ikarashi. 1986. A model of snow glide acceleration to full depth avalanche release. Rep. Natl. Res. Cent. Disaster Prevention, 38, 169-180. [In Japanese.]

Quervain, M.R. de. 1966. Problem of avalanche research. International Association of Scientific Hydrology Publication 69 (Symposium at Davos 1965 - Avalanches and Physics of Snow), 15-22.

Salm, B. 1977. Snow forces. J. Glaciol., 19(81), 67-100.

Tusima, K. 1977. Studies of friction on single crystals of ice I. - Load, velocity and temperature effects on friction between steel ball and (0001) and (0110) of ice, and adhesion theory as a mechanism of friction. Low. Temp. Sci., Ser. A, 35, 1-22. [In Japanese.] 